INPLASY

PROTOCOL

To cite: Moraschini et al. The role of dental implant location on the prevalence of periimplantitis: a systematic review and meta-analysis. Inplasy protocol 202140101. doi: 10.37766/inplasy2021.4.0101

Received: 19 April 2021

Published: 19 April 2021

Corresponding author: Monica Calasans-Maia

monicacalasansmaia@gmail.com

Author Affiliation:

Oral Surgery Department Universidade Federal

Fluminense

Support: None.

Review Stage at time of this submission: Formal screening of search results against eligibility criteria.

Conflicts of interest:

None declared.

\section{The role of dental implant location on the prevalence of peri-implantitis: a systematic review and meta-analysis}

Moraschini, V1; Sartoretto, SC2; Kischinhevsky, IC3; Correa-Silva, VG4; Calasans-Maia, MD5 .

Review question / Objective: Which intra-oral region has the highest risk for peri-implantitis in patients with dental implants in occlusal function with at least one year of function?

Condition being studied: This SR aims to evaluate (I) the prevalence of peri-implantitis in at least six months of implant placement and (II) the correlation with the type of prosthetic retention and the anatomical implant sites.

Information sources: PubMed/MEDLINE, the Cochrane Central Register of Controlled Trials, Scopus, and Lilacs were used to search for articles that were published prior to March 2021, without any restrictions regarding date or language. A search of the gray literature using the Literature Report and OpenGrey databases was also conducted. Finally, the study reference lists were evaluated (cross-referenced) to identify other potential studies for inclusion.

INPLASY registration number: This protocol was registered with the International Platform of Registered Systematic Review and Meta-Analysis Protocols (INPLASY) on 19 April 2021 and was last updated on 19 April 2021 (registration number INPLASY202140101).

\title{
INTRODUCTION
}

Review question / Objective: Which intraoral region has the highest risk for periimplantitis in patients with dental implants in occlusal function with at least one year of function?
Rationale: Peri-implant diseases are initially infectious triggered by an inflammatory response to biofilm collection, as the main factor of its pathogenesis similar to periodontitis, a series of conditions can increase their risk. Among them, patient- 
and implant-related factors as smoking, systemic diseases such as diabetes mellitus, periodontal disease, and poor oral hygiene have been assumed in many studies. Additional factors are cited in the literature as a possible risk to the development of peri-implant bone loss, including the prothesis-related factors. Some studies have reported a relationship between cement-retained restorations and periimplantitis, which was likely due to the presence of residual cement in the sulcus. Also, implant-related factors, as anatomic implant localization (anterior/posterior, maxilla, or mandible), are suggested as a potential risk to the development of periimplant bone loss. Although the literature shows a tendency to the higher prevalence in mandibular implants, this correlation is not a consensus in the literature. Overall, the prevalence of peri-implantitis has been evaluated in several clinical studies with different follow-ups. In a previous systematic review (SR) that assessed the prevalence, extent, and severity of periimplant diseases, 11 studies were evaluated. The prevalence of peri-implant mucositis and peri-implantitis was $43 \%$ (ranged from 19 to $65 \%$ ) and $22 \%$ (1 to $47 \%$ ), respectively. Nevertheless, these results should be interpreted with caution since there are differences between the case definitions, follow-up times, and patients' selection in the studies assessed.

Condition being studied: This SR aims to evaluate (I) the prevalence of periimplantitis in at least six months of implant placement and (II) the correlation with the type of prosthetic retention and the anatomical implant sites.

\section{METHODS}

Search strategy: PubMed/MEDLINE, the Cochrane Central Register of Controlled Trials, Scopus, and Lilacs were used to search for articles that were published prior to March 2021, without any restrictions regarding date or language. A search of the gray literature using the Literature Report and OpenGrey databases was also conducted. Finally, the study reference lists were evaluated (cross- referenced) to identify other potential studies for inclusion.

Participant or population: Patients who have had dental implants in occlusal function for at least one year.

Intervention: Dental implants.

Comparator: Prevalence of peri-implantitis in dental implants placed in the maxilla vs. mandible. Prevalence of peri-implantitis in dental implants placed in the anterior vs. posterior regions.

Study designs to be included: Observational cohort studies (prospective or retrospective), cross-sectional, and case controls.

Eligibility criteria: The main question and the eligibility criteria were elaborated through the PICOS strategy. The search and selection process for studies was conducted by two independent authors. First, titles and abstracts were analyzed. After, pre-selected studies were read in full to verify that they met all eligibility criteria. The search concordance between the two reviewers was evaluated by Cohen's Kappa (k) test. Disagreements between the reviewing authors were resolved through careful discussion. The following eligibility criteria have been established: Population: patients who have had dental implants in occlusal function for at least one year. Intervention: dental implants Comparison: prevalence of peri-implantitis in dental implants placed in the maxilla vs. mandible. Prevalence of peri-implantitis in dental implants placed in the anterior vs. posterior regions. Outcome: Prevalence of periimplantitis. Study design: Observational cohort studies (prospective or retrospective), cross-sectional, and case controls. The exclusion criteria included animal studies, in vitro studies, case series, case reports, and reviews. In addition, studies that did not report the regions of occurrence of peri-implantitis and with follow-up of less than one year, were excluded. 
Information sources: PubMed/MEDLINE, the Cochrane Central Register of Controlled Trials, Scopus, and Lilacs were used to search for articles that were published prior to March 2021, without any restrictions regarding date or language. A search of the gray literature using the Literature Report and OpenGrey databases was also conducted. Finally, the study reference lists were evaluated (crossreferenced) to identify other potential studies for inclusion.

Main outcome(s): Main Outcome: Prevalence of peri-implantitis. The dichotomous variable (prevalence of periimplantitis) of the included studies were categorized in subgroups, depending on intra-oral location, and analyzed in a metaanalysis of all implant levels.

\section{Additional outcome(s): No additional outcomes were evaluated.}

Data management: Two investigators (I.K. and S.S.) independently extracted data from included studies based on the predefined standardized form of data collection. Any discrepancies between two investigators in extracted data were solved by a third expert investigator through discussion (V.M.F.). Extracted information includes study basic characteristics (including title, first author, et al), Studies characteristics (Study Design Follow-up Number of patients), Total number of implants / peri-implantits (\%), Implant location/Periimplatits (total / events), Type of prosthesis / peri-implantits ( $n, \%)$, Prosthesis retention / peri-implantits (n, \%), History of periodontitis (patient-level) / peri-implantitis, Smokers (patient-level) / peri-implantitis, Diabetes (patient-level) / peri-implantitis.

Quality assessment / Risk of bias analysis: Study methodological quality will be measured using the NIH Quality Assessment tool for observational cohort and cross-sectional studies for risk of bias through selection, performance, detection, attrition, reporting and other biases. Fourteen items are further assessed as Good, Fair, or Poor risk of bias. Two independent reviewers will assess the methodological quality for all included studies, respectively. A third expert investigator will help to solve all disagreements that occur between two investigators.

Strategy of data synthesis: The study data were extracted and systematically reviewed when available, the following data were obtained from the studies: authors, study design, length of follow-up, number of patients, number of implants, the prevalence of peri-implantitis, implant location, type of prosthesis, type of prosthesis retention, history of periodontitis, and number of smokers and diabetics.

Subgroup analysis: According to the localization of the installed implants the subgroups are: anterior maxilla; posterior maxilla; anterior mandible; and posterior mandible.

Sensitivity analysis: If sufficient available data are extracted, we will plan to conduct sensitivity analysis to check the stability for the outcome results by excluding low methodological quality studies.

Language: English.

Country(ies) involved: Brazil.

Other relevant information: Not applicable.

Keywords: Peri-implantitis prevalence, dental implants, implant site.

Dissemination plans: The authors will submit this study to a peer-reviewed journal for publication.

Contributions of each author:

Author 1 - Vittorio Moraschini Conceptualization, Methodology, Software, Formal Analysis, Data Curation, WritingOriginal Draft Preparation, Writing-Review, and Editing, Visualization and Project Administration.

Email: vitt.mf@gmail.com

Author 2 - Suelen Sartoretto Conceptualization, Methodology, Software, 
Investigation, Data Curation, WritingOriginal Draft Preparation, Writing-Review and Editing, and Visualization.

Email: susartoretto@hotmail.com

Author 3 - Ingrid Kischinhevsky Conceptualization, Methodology, Validation, Formal Analysis, Investigation, Data Curation, Visualization, and Supervision.

Email: ingrid.chaves@hotmail.com

Author 4 - Monica Calasans-Maia Conceptualization, Writing-original draft writing-review and editing, Visualization, Supervision, and Project Administration.

Email: moniicacalasansmaia@gmail.com

Author 5 - Vinicius Correa-Silva Conceptualization, Resources, Writingoriginal draft writing-review and editing, Visualization, Supervision, and Project administration.

Email: viniciusgcs@hotmail.com 